\title{
Potential for Reducing Time to Referral for Colorectal Cancer Patients in Primary Care
}

\author{
Nicole F. van Erp \\ Charles W. Helsper \\ Saskia M. Olyboek \\ Ramon R. T. Janssen \\ Amber Winsveen \\ Petra H. M. Peeters \\ Niek J. de Wit \\ Julius Centre for Health Sciences and \\ Primary Care, University Medical Centre \\ Utrecht, Utrecht University, Utrecht, The \\ Netherlands
}

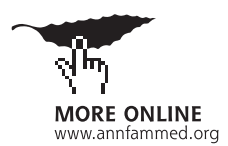

Conflicts of interest: authors report none.

\section{CORRESPONDING AUTHOR}

Charles Helsper, MD, MSc, PhD

Julius Centre for Health Sciences and Primary Care

University Medical Centre Utrecht P.O. Box 85500

3508 GA Utrecht, The Netherlands

C.W.Helsper-2@umcutrecht.nl

\begin{abstract}
PURPOSE An optimal diagnostic process in primary care is pivotal for reducing cancer-related disease burden. This study aims to explore reasons for long times to referral for Dutch colorectal cancer (CRC) patients in primary care.
\end{abstract}

METHODS A retrospective cohort study of anonymized free-text primary care records from the Julius General Practitioners' Network database, linked to the Netherlands Cancer Registry. Patients with a confirmed CRC diagnosis from 2007 through 2011 that symptomatically presented in primary care were included. Median time and interquartile ranges from presentation in primary care to referral were calculated for multiple patient and presentation characteristics. Associations of these characteristics with long time to referral (75th percentile was $\geq 59$ days) were examined with log-binomial regression analyses. Routes to referral of patients with the longest times to referral were explored using thematic free-text analyses (90th percentile at $\geq 219$ days).

RESULTS Among the 309 people with CRC, patients who were female, did not have a registered family history, had a history of malignancy, lacked alarm symptoms at presentation, or had hemorrhoids at physical examination were at risk for longer time to referral in univariable analyses (longer median durations and/ or univariable association with the 75th percentile). Only presentation without alarm symptoms showed a statistically significant association with long duration (75th percentile) in multivariable analysis (relative risk $=1.7 ; 95 \% \mathrm{Cl}, 1.1-2.6$ ). Thematic exploration of the diagnostic routes to referral of patients with the longest durations (90th percentile) showed 2 dominating themes: "alternative working diagnosis" and "suboptimal diagnostic strategies," and included the subthemes "omitting to reconsider an initial diagnosis" and "lacking follow-up."

CONCLUSIONS Long time to referral for CRC in primary care is mainly related to low cancer suspicion. There is potential for reducing the longest times to referral for patients with CRC in primary care, with earlier reconsideration of the initial hypothesis and implementation of strict follow-up consultations.

Ann Fam Med 2019;17:419-427. https://doi.org/10.1370/afm.2446.

\section{INTRODUCTION}

olorectal cancer (CRC) is the second most common cancer and the second most common cause of cancer-related death in Europe, with approximately 450,000 new patients with CRC and 215,000 CRC-related deaths annually. ${ }^{1}$ Prognosis for colorectal cancer is mainly dependent on the tumor stage at diagnosis., ${ }^{2,3}$ Prompt referral and diagnosis are considered important for improved clinical outcomes. ${ }^{4,5}$

Much effort has already been applied to optimize early detection of bowel cancer with implementation of population screening in most European countries. ${ }^{6}$ However, in primary care-based health care systems, in which the general practitioner (GP) is the patient's first contact and triages the patient's further access to the system, most CRC patients present to a GP with symptoms. Therefore, timely recognition of cancer-related complaints and adequate referral by the GP are and will remain essential to reduce time to diagnosis. 
In the United Kingdom and the Netherlands, the current median time from first consultation with cancer-related complaints to referral is approximately 1 week for CRC patients. ${ }^{78}$ Although this median duration seems acceptable, time to referral varies greatly, with durations of months and even years, for $10 \%$ to $25 \%$ of CRC patients. ${ }^{7,8}$

Several studies have reported factors associated with diagnostic delay in CRC patients. They included explanations for doctor's delay (eg, initial misdiagnosis, inadequate examination, inaccurate investigations) and found that older patients and those with comorbid conditions were at increased risk of delay. ${ }^{9-13}$

These studies, however, were often limited to analyses of coded research data, strictly quantitative analyses, and/or lacked opportunities to link determinants of delayed referral to actual time spent in primary care. Therefore, explanations for suboptimal referral from these studies may be incomplete and oversimplified.

The aim of the current study is to perform a more detailed assessment of the time from presentation in primary care to referral for patients with CRC, including the identification of mechanisms causing long times to referral.

\section{METHODS}

\section{Study Design and Data Source}

A retrospective cohort study was performed using free text and coded routine primary care data from the Julius General Practitioner's Network (JGPN) database, linked to data in the Netherlands Cancer Registry. The JGPN database contains nonreducible free text and coded information from primary care electronic health records of over 300,000 patients from a central region of the Netherlands. ${ }^{14}$ The Netherlands Cancer Registry provides reliable and detailed information on Dutch cancer patients since 1989. A more extensive description of study design and data sources is provided elsewhere. ${ }^{7}$

\section{Patient Selection}

Patients, aged 20 through 90 years, were selected if they were registered with International Classification of Primary Care version 1 (ICPC-1) code D75 (for malignant neoplasm colon/rectum) in the JGPN database from 2007 through 2011, and were registered with the same diagnosis in the Netherlands Cancer Registry, validating diagnoses.

Only patients who initially visited the GP with complaints or symptoms directly or indirectly related to CRC, and were referred by the GP, were included. Patients with substantially missing information or unclear electronic health records contents were excluded.

\section{Data Collection}

Primary Care Interval

The primary care interval was defined according to the Aarhus statement, ${ }_{1}^{15}$ as the period of time from first consultation with cancer-related signs and/or symptoms in primary care to referral to secondary care. Date of first consultation was defined as the first contact with the GP (in person or by telephone) with colorectal cancer-related signs or symptoms. For patients with vague or nonspecific signs or symptoms, the first consultation with the complaints that eventually led to the CRC diagnosis and could reasonably be related to the cancer, was chosen.

Date of referral was defined as the moment the responsibility for the patient was transferred from GP to secondary care, that is, the day on which the GP decided to refer and sent a referral letter. Referral to radiology or endoscopy departments for imaging was used as the date of referral, if abnormal findings subsequently lead to referral to a specialist, without further involvement of the GP. In case of multiple referrals to, or cross-referrals in secondary care, the first referral for further exploration of cancer-related symptoms was chosen.

The free text and coded primary care electronic health records data of all symptomatic CRC patients were studied by 2 researchers (R.J, A.W.) from 5 years before the date of diagnosis as registered in the Netherlands Cancer Registry data to determine the occurrence of the first colorectal cancer-related signs or symptoms and date of referral. Five years before 1 year after diagnosis was arbitrarily chosen to ensure a comprehensive overview of the complete diagnostic process, including the onset of CRC symptoms and other relevant morbidities.

When there was doubt about date of first presentation and/or referral, diagnostic paths were discussed with a second researcher or with the complete research team (N.vE., S.O., R.J., A.W., C.H.) until consensus was reached.

\section{Characteristics}

The decision to collect data for certain characteristics and to include them in our initial, univariable analyses was based on previously reported predictors in the literature (eg, comorbidity, including psychiatry), on clinical relevance of patient and disease characteristics, and on availability of data in the JGPN registry. ${ }^{9-13}$ Disease, patient, and presentation characteristics that could be extracted are summarized in Table 1 and Table 2. Elaborate descriptions of the characteristics and collection methods are provided in Supplemental Table 1, available at http://www.AnnFamMed.org/content/17/5/419/ suppl/DC1/. 


\section{Analyses}

Primary Care Interval by Characteristic

The length of the primary care interval was previously reported for the total population (median 8 days; interquartile range $[\mathrm{IQR}]=1-59$; range, $1-1,177)^{7}$ and is now calculated for multiple patient and presentation characteristics. Durations are reported as medians, interquartile ranges, and 90th percentiles, because of the substantially right-skewed distribution of the data. ${ }^{7}$ Differences in median durations between categories of characteristics were tested for statistical significance using the Mann-Whitney $U$ test for characteristics with 2 categories and the Kruskal-Wallis test for characteristics with 3 or more categories.

\section{Determinants of Long Duration}

Long duration primary care interval was defined as periods of time greater than or equal to the 75 th percentile ( $\geq 59$ days). Uni- and multivariable log-binomial regression analyses were performed to identify characteristics associated with long duration. Characteristics that were significantly associated with long duration $(P$ $<.05)$ in univariable analysis were included in multivariable analysis.

\section{Thematic Analyses for the Longest Duration}

Longest duration primary care interval was defined as periods of time greater than or equal to the 90th percentile ( $\geq 219$ days). Mechanisms leading to the longest primary care intervals were extracted from verbatim transcriptions of the free-text registrations of all consultations preceding referral. The diagnostic route to referral and the deliberations of the GP were analyzed using open coding, axial coding, and selective coding. Details of the qualitative data analysis are available in the Supplemental Appendix, available at http://www. AnnFamMed.org/content/17/5/419/suppl/DC1/.

\section{Software}

Data collection, transformation, and analyses were performed in SPSS version 22.0 (SPSS Inc).

\section{RESULTS}

\section{Patients}

Of 416 patients with a validated CRC diagnosis identified in the JGPN database, 320 initially presented in primary care, of whom 313 were diagnosed after symptomatic presentation. The referral date was available for 309. The remaining 107 patients were excluded for reasons shown in Figure 1. Included CRC patients had a mean age of 66.7 years with a standard deviation of 12.2 years and $154(49.8 \%)$ were female. Table 1 and Table 2 show the characteristics of CRC patients included in this study.

\section{Primary Care Interval by Characteristic}

Table 2 shows median and IQR data for duration of primary care intervals calculated for patient and presentation characteristics. Characteristics with a statistically significant difference in median duration were: sex, 13 days for female patients vs 4 days for male patients; registered family history of CRC, 11 days for patients without a registered family history of CRC vs 1 day for those with registered history; and presentation with only nonalarming gastrointestinal symptoms, 26 days compared to 2 days for those with alarming gastrointestinal signs. For patients diagnosed with stage IV colorectal cancer, median duration of the primary care interval was 23 days, which is more than 2 times longer than for patients with less advanced disease stages.

Although not statistically significant, duration of primary care intervals were more than 3 weeks longer for patients aged under 50 years, patients presenting with psychiatric comorbidity (mostly depression and anxiety), and patients with hemorrhoids at physical examination.

\section{Determinants of Long Duration}

In univariable log-binomial analysis the following characteristics (Table 2) were significantly associated with long duration primary care intervals (75th percentile, $\geq 59$ days): female sex, a history of malignancy, presentation with nonalarming gastrointestinal symptoms, and presence of hemorrhoids. Multivariable analyses showed a statistically significant association with long duration primary care interval for presentation with nonalarming gastrointestinal symptoms.

\section{Thematic Analysis of Longest Duration}

The longest duration of primary care intervals $\left(90^{\text {th }}\right.$ percentile, $\geq 219$ days) was seen in 31 patients, with
Table 1. Disease Characteristics of Symptomatic CRC Patients Referred by GP $(\mathrm{N}=309)$

\begin{tabular}{lc:|lll}
\hline Characteristic & No. (\%) & & Characteristic & No. (\%) \\
\cline { 1 - 2 } TNM stage at diagnosis & & & Tumor location & \\
O & $1(0.3)$ & & Proximal colon & $90(29.1)$ \\
I & $41(13.3)$ & & Distal colon & $20(6.5)$ \\
II & $83(26.9)$ & & (Recto)sigmoid & $99(32.0)$ \\
III & $114(36.9)$ & & Rectal & $97(31.4)$ \\
IV & $65(21.0)$ & & Colon unspecified & $3(1.0)$ \\
Unknown & $5(1.6)$ & & & \\
\hline CRC = colorectal cancer; GP = general practitioner; TNM = tumor, nodes, metastases.
\end{tabular}


Table 2. Patient and Presentation Characteristics of Symptomatic CRC Patients, Duration of Primary Care Interval, and Log-Binomial Regression Analysis for 75th Percentile $(\mathrm{N}=309)$

\begin{tabular}{|c|c|c|c|c|c|c|}
\hline Characteristic & No. & $\begin{array}{l}\text { Duration, d, } \\
\text { Median (IQR) }\end{array}$ & $P$ Value $^{a}$ & $\mathrm{P}^{\mathrm{b}} \mathrm{b}$ & $\begin{array}{c}\text { Univariable RR } \\
(95 \% \mathrm{Cl})\end{array}$ & $\begin{array}{c}\text { Multivariable RR } \\
(95 \% \mathrm{Cl})\end{array}$ \\
\hline \multicolumn{7}{|l|}{ Age, y } \\
\hline$\leq 50$ & 35 & $34(1-233)$ & & 491 & $1.5(0.8-3.0)$ & \\
\hline $51-60$ & 47 & $3(1-15)$ & & 408 & $0.6(0.3-1.4)$ & \\
\hline $61-70$ & 100 & $14(1-47)$ & & 94 & $0.8(0.4-1.4)$ & \\
\hline $71-80$ & 91 & $6(1-61)$ & & 204 & $0.9(0.5-1.7)$ & \\
\hline $81-90$ & 36 & $8(1-68)$ & .154 & 150 & 1 (ref) & \\
\hline \multicolumn{7}{|l|}{ Sex } \\
\hline Male & 155 & $4(1-47)$ & & 101 & 1 (ref) & 1 (ref) \\
\hline Female & 154 & $13(1-78)$ & .004 & 321 & $1.6(1.1-2.4)$ & $1.4(0.9-2.1)$ \\
\hline \multicolumn{7}{|l|}{ SES $2010^{c}$} \\
\hline Low & 81 & $12(1-72)$ & & 240 & 1 (ref) & \\
\hline Medium-low & 79 & $9(1-63)$ & & 239 & $0.9(0.5-1.5)$ & \\
\hline Medium-high & 73 & $7(1-51)$ & & 118 & $0.7(0.4-1.3)$ & \\
\hline High & 76 & $6(1-47)$ & .551 & 223 & $0.7(0.4-1.2)$ & \\
\hline \multicolumn{7}{|c|}{ Registered comorbidity } \\
\hline \multicolumn{7}{|l|}{ Chronic somatic } \\
\hline No & 62 & $5(1-48)$ & & 326 & 1 (ref) & \\
\hline Yes & 247 & $10(1-61)$ & .317 & 198 & $1.1(0.7-1.9)$ & \\
\hline$\geq 2$ & 181 & $9(1-58)$ & & 203 & $1.1(0.6-1.8)$ & \\
\hline$\geq 4$ & 70 & $8(1-43)$ & & 98 & $0.9(0.5-1.7)$ & \\
\hline \multicolumn{7}{|l|}{ Gastrointestinal } \\
\hline No & 256 & $8(1-63)$ & & 219 & 1 (ref) & \\
\hline Yes & 53 & $15(2-48)$ & .622 & 119 & $0.7(0.4-1.3)$ & \\
\hline \multicolumn{7}{|l|}{ Psychiatric } \\
\hline No & 290 & $8(1-58)$ & & 204 & 1 (ref) & \\
\hline Yes & 19 & $22(2-84)$ & .203 & 538 & $1.1(0.5-2.3)$ & \\
\hline \multicolumn{7}{|c|}{ Registered family history of CRC } \\
\hline Not registered & 267 & $11(1-65)$ & & 233 & 1 (ref) & \\
\hline Negative & 30 & $1(1-13)$ & & 87 & $0.5(0.2-1.3)$ & \\
\hline Positive & 12 & $2(1-34)$ & .003 & 87 & $0.3(0.1-2.0)$ & \\
\hline \multicolumn{7}{|c|}{ Consultation frequency for year prior to first CRC consultation } \\
\hline$\leq 2$ & 56 & $2(1-29)$ & & 117 & 1 (ref) & \\
\hline $3-11$ & 188 & $11(1-64)$ & & 235 & $1.4(0.8-2.5)$ & \\
\hline$\geq 12$ & 65 & $12(1-54)$ & .093 & 120 & $1.2(0.6-2.3)$ & \\
\hline \multicolumn{7}{|c|}{ History of malignancy } \\
\hline No & 267 & $7(1-50)$ & & 219 & 1 (ref) & 1 (ref) \\
\hline \multirow[t]{2}{*}{ Yes } & 42 & $18(2-84)$ & .101 & 178 & $1.7(1.1-2.6)$ & $1.5(0.9-2.2)$ \\
\hline & & & & & & continues \\
\hline
\end{tabular}

durations up to 1,177 days. The majority of these patients were female $(n=22,71 \%)$ as opposed to $47.5 \%$ female patients with a duration less than 219 days $\left(\chi^{2}\right.$ test $P=.013$ ). Mean age was 60.4 years (standard deviation $=15.5$ ) compared with patients aged 67.4 years (standard deviation $=11.6$ ) for those with a duration less than 219 days ( $t$-test $P=.020)$. Of these patients, $71 \%$ were diagnosed with stage III or IV colorectal cancer, compared with $57 \%$ of patients with a duration less than 219 days $\left(\chi^{2}\right.$ test $\left.P=.121\right)$. The number of cancer-related consultations during the primary care interval for these patients ranged from 2 to 25 . All factors associated with longest duration primary care interval are shown in Figure 2.

The reasons for longest duration intervals were generally multifactorial. Several themes were related to postponed referral, but the dominant theme was "having an alternative working diagnosis," with a leading subtheme "the presence of an explanatory concomitant condition." The second main theme was "suboptimal diagnostic strategies," with subthemes "omitting to reconsider an initial diagnosis" and "lacking follow-up."

In all 31 patients the GP had "an alternative working diagnosis" that was not colorectal cancer. The 
Table 2. Patient and Presentation Characteristics of Symptomatic CRC Patients, Duration of Primary Care Interval, and Log-Binomial Regression Analysis for 75th Percentile $(\mathbf{N}=309)$ (continued)

\begin{tabular}{|c|c|c|c|c|c|c|}
\hline Characteristic & No. & $\begin{array}{l}\text { Duration, d, } \\
\text { Median (IQR) }\end{array}$ & $P$ Value & P90 & $\begin{array}{l}\text { Univariable RR } \\
\qquad(95 \% \mathrm{CI})\end{array}$ & $\begin{array}{l}\text { Multivariable RR } \\
(95 \% \mathrm{CI})\end{array}$ \\
\hline \multicolumn{7}{|c|}{ Main registered symptom at first CRC consultation ${ }^{f}$} \\
\hline Alarm Gl symptom(s) & 168 & $2(1-28)$ & & 123 & 1 (ref) & 1 (ref) \\
\hline Nonalarm Gl symptom(s) & 113 & $26(5-87)$ & & 257 & $1.9(1.2-2.8)$ & $1.7(1.1-2.6)$ \\
\hline Other symptom(s) & 28 & $13(2-43)$ & .000 & 273 & $0.9(0.4-2.2)$ & $0.9(0.4-2.1)$ \\
\hline No & 298 & $8(1-54)$ & & 219 & 1 (ref) & \\
\hline Yes & 11 & $69(1-115)$ & .192 & 213 & $2.3(1.3-4.1)$ & \\
\hline \multicolumn{7}{|l|}{ TNM stage at diagnosis } \\
\hline 0 & 1 & 87 & & $\ldots$ & $\ldots$ & \\
\hline I & 41 & $2(1-42)$ & & 83 & 1 (ref) & \\
\hline II & 83 & $7(1-48)$ & & 213 & $1.1(0.5-2.3)$ & \\
\hline IV & 65 & $23(3-92)$ & & 502 & $1.9(0.9-3.8)$ & \\
\hline Unknown & 5 & $5(1-246)$ & .013 & $\cdots$ & $\cdots$ & \\
\hline \multirow{2}{*}{\multicolumn{7}{|c|}{ 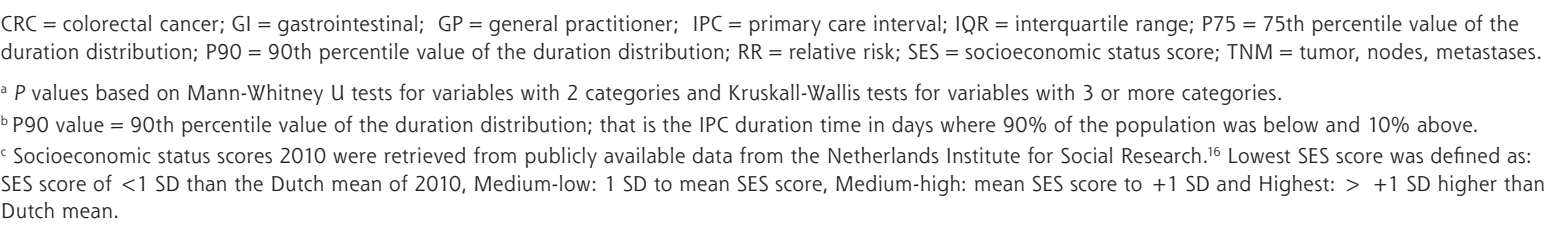 }} \\
\hline & & & & & & \\
\hline \multicolumn{7}{|c|}{$\begin{array}{l}\text { d Chronic somatic comorbidities were defined according to O'Halloran et al. }{ }^{17} \text { Gastrointestinal comorbidities were all relevant GI-related registered comorbidities or } \\
\text { conditions in either episode list or mentioned during GP consultations: irritable bowel syndrome, reflux disease, esophagitis, dyspepsia, abdominal pain, peptic ulcer, } \\
\text { hiatus or abdominal hernia, benign GI neoplasms/polyps, constipation, chronic diarrhea, cholelithiasis, diverticulosis, anal fissures. Psychiatric comorbidities were all } \\
\text { chronic psychiatric comorbidities according to O'Halloran et al. }{ }^{17}\end{array}$} \\
\hline \multicolumn{7}{|c|}{ e Registered occurrence of colorectal cancer in a first degree family member. } \\
\hline \multicolumn{7}{|c|}{$\begin{array}{l}\text { 'Alarm symptoms for colorectal cancer were defined as rectal blood loss, unintended weight loss, anemia, and a palpable tumor. Gl symptoms include all GI-related, } \\
\text { nonalarming symptoms. Other symptoms are all remaining, nonalarming, non-Gl symptoms. }\end{array}$} \\
\hline
\end{tabular}

factor most often explaining the occurrence of an alternative working diagnosis was subtheme "presence of an explanatory concomitant condition," either preexisting or detected during consultation. Conditions included hemorrhoids or fissures in cases of rectal blood loss; hypermenorrhagia in cases of anemia; and inflammatory bowel disease, poorly regulated hypothyroidism, psychological conditions or stress, alcohol or drug abuse, in cases of several other cancer-related complaints. Other subthemes causing the GP to stick to the original hypothesis were: "good symptomatic response to initial therapy," such as laxatives for constipation, antacids for gastric complaints, and mesalazine for inflammatory bowel disease, and "misleading results from additional testing," including negative gastroscopy or ultrasound, identification of a pathogen in stool culture, absence of anemia or inflammatory markers in blood test results, and "intermittent character of the complaints."

Second, GPs and/or patients sometimes followed "suboptimal diagnostic strategies." This included "inadequate follow up," including later consultations postponed by patients without clear reason and patients initially unwilling to undergo further investigation. The second subtheme that could be derived was "the GP omitting to reconsider the initial diagnosis." That is, the GP did no further investigating anemia or rectal blood loss, even after the initial explanatory cause had resolved.

\section{DISCUSSSION}

\section{Summary}

Symptomatic colorectal cancer patients presenting to primary care were at risk for longer time to referral if they had patient and presentation characteristics that lowered cancer suspicion. Included (by univariable analysis) were patients who were female, did not have a registered family history, did not have alarm symptoms, had a history of malignancy, and had hemorrhoids at physical examination. Thematic exploration of the diagnostic routes to referral of patients with longest durations (90th percentile) showed 2 dominating themes: "alternative working diagnosis" and "suboptimal diagnostic strategies," including subthemes "omitting to reconsider an initial diagnosis" and "lack- 


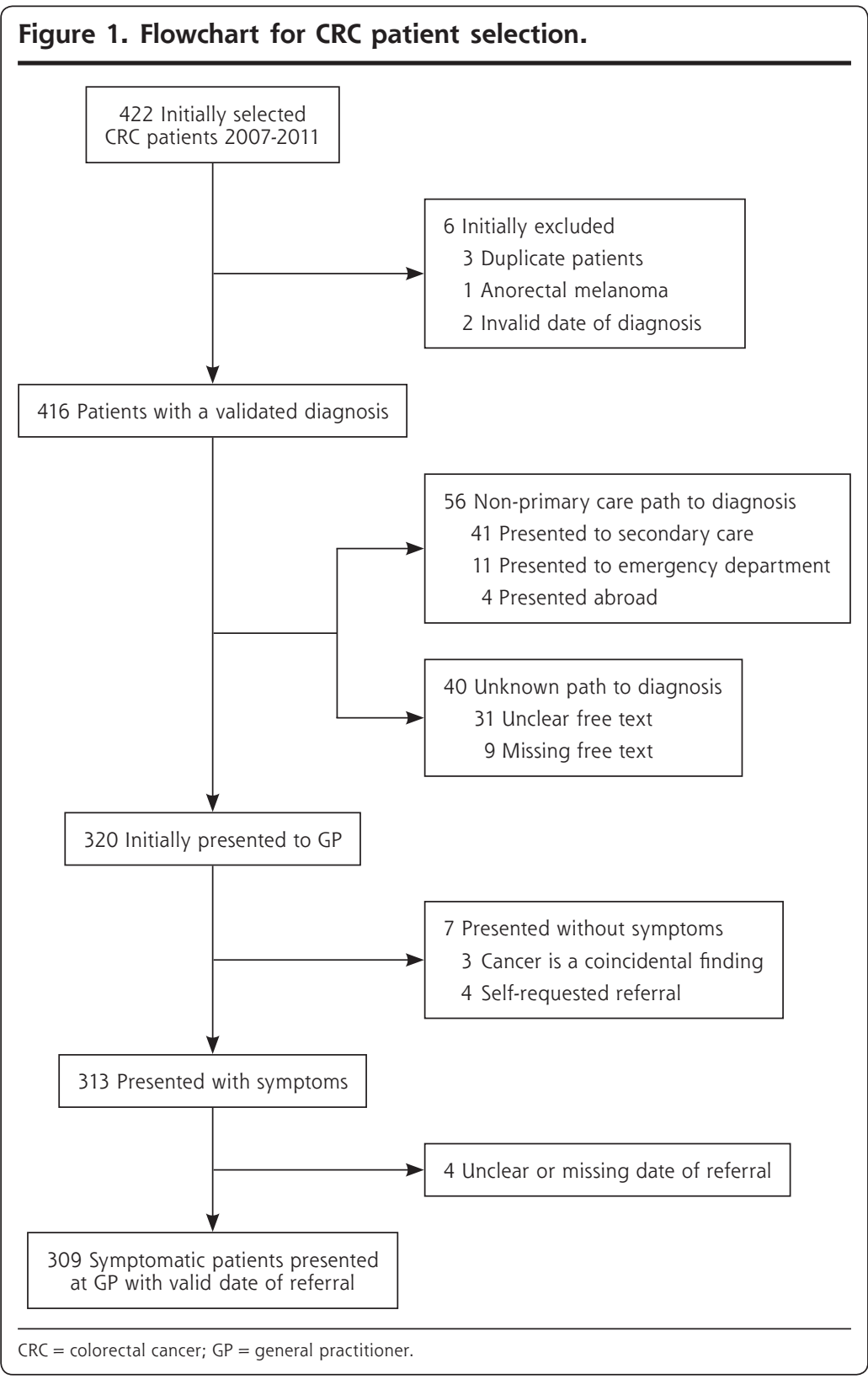

tial sample sizes and therefore the strength of inferences.

Limitations also include the need for interpretation and potential incompleteness of routine care data. The main challenge is to retrospectively identify the first consultation with cancer-related complaints, particularly in patients with less specific symptom presentations. The risk of misattribution of symptoms to cancer was minimized in our study by discussing doubtful cases with a team of researchers with primary care experience. Nevertheless, consequential over- or underestimation of time to referral may have occurred. Furthermore, for $10 \%$ of the initially selected patients with validated CRC diagnosis, the diagnostic path was too unclear to determine eligibility and/or date of first presentation. Lack of clarity about the diagnostic path is most likely due to incomplete GP registrations or initial presentation to secondary care providers. Since cancer patients symptomatically presenting to primary care (our population of interest) are unlikely to be subject to incomplete GP registration, we expect that for the great majority of patients with unclear diagnostic paths, secondary care presentations are the most plausible explanation. A final limitation may be that, when focusing on delay in the diagnostic path of CRC, including only the primary care interval provides an incomplete scope. ${ }^{7}$ There may already be delay before presentation at the GP, as well as delay after referral to secondary care. Patient, population, specialist, and system causes may all contribute to delay in the pre- and post-primary care intervals.

\section{Comparison With Existing Literature}

The increased primary care intervals for patients aged under 50 years and female patients are consistent with international literature. ${ }^{9,10}$ This is probably related to the fact that colorectal cancer occurs more often in older age groups and male patients. ${ }^{19}$ Pain and bleeding are associated with prompt referral according to the literature. ${ }^{18}$ Bleeding aligns with our finding that patients with alarm symptoms, although not assessed for dif- 


\section{Figure 2. Factors contributing to longest duration ( $\geq 219$ days) for symptomatic CRC patients. ${ }^{\mathrm{a}}$}

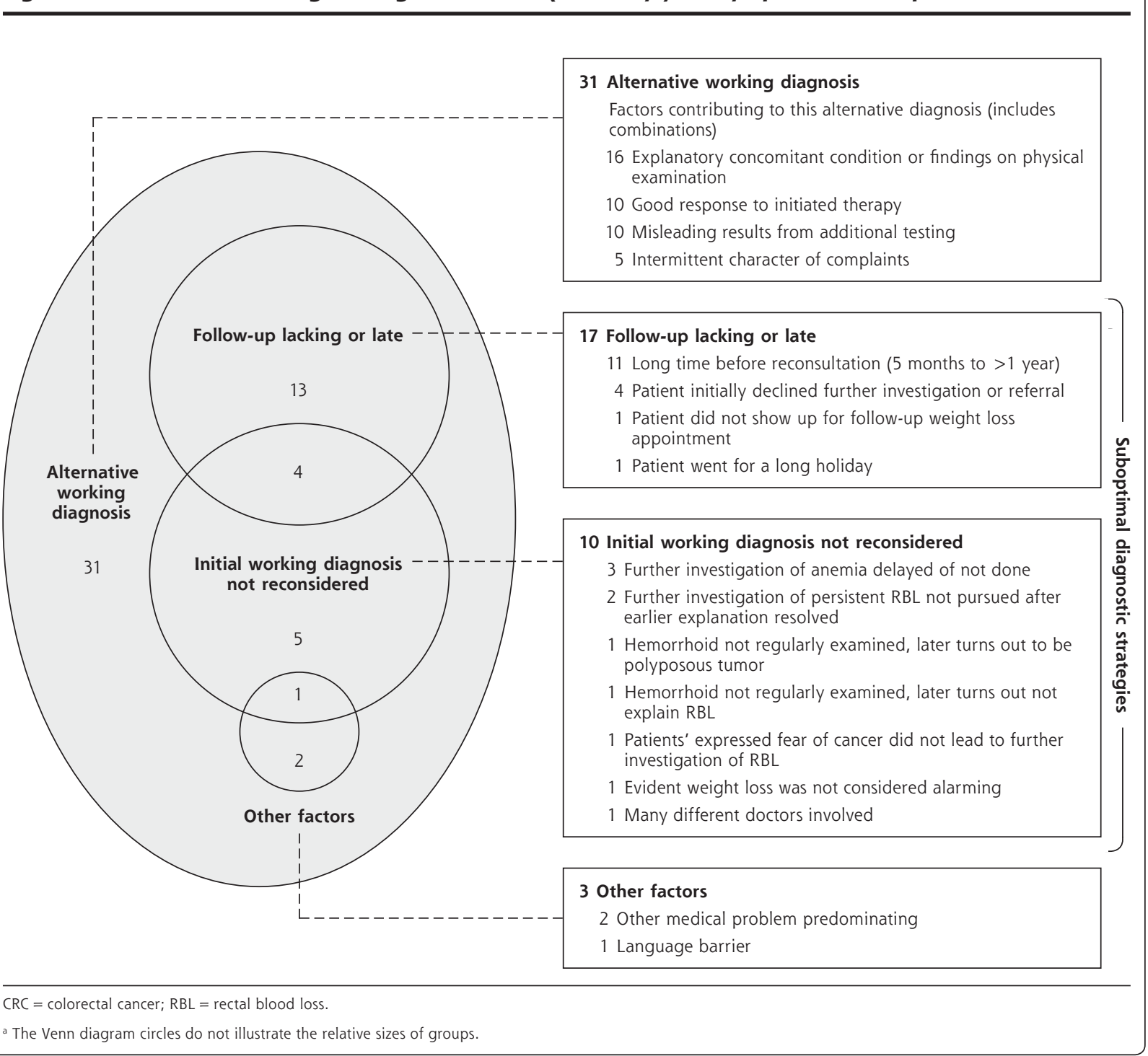

ferent alarm symptoms separately, had shorter primary care intervals, and patients with less specific gastrointestinal complaints had higher risk of delayed referral.

In our earlier study, psychiatric comorbidity was found to be associated with GP delay. ${ }^{13}$ Although the univariable association of psychiatric comorbidity with long duration (75th percentile) primary care intervals in this study did not reach statistical significance, the median primary care interval was 2 weeks longer for patients with psychiatric comorbidity. One explanation for this association is that comorbid conditions compete for clinical attention and may provide alternative explanations for cancer-related symptoms. ${ }^{12}$ This was also reflected by the longer median durations we found for patients with gastrointestinal comorbidity.
Consistent with the alternative explanation argument, we found that the patients with hemorrhoids had substantially longer median times to referral and long duration in the univariable analysis. Comparably, the broader concept of "initial misdiagnosis" was associated with practitioner delay in over $75 \%$ of earlier studies that assessed this factor. " Having an alternative diagnosis" was also the main factor contributing to longest durations (90th percentile) in the thematic analysis in the present study.

Our thematic analysis, based on extensive free-text inquiry, adds to the previous knowledge that reasons for substantial delay are often multifactorial. We identified "suboptimal diagnostic strategies" as the second main theme, with subthemes "omitting to reconsider an initial diagnosis" and "lacking follow-up." These 
latter 2 subthemes leave room for improvement in the diagnostic process in primary care by preventing unnecessary delay.

The extent to which reduction of the primary care interval duration could contribute to improved clinical outcomes is uncertain. A recent study by Tørring et al underlined the complexity of this association.

Longer primary care intervals appeared to increase the odds of advanced CRC, but with even longer intervals the odds decreased again. ${ }^{4}$ We found longer primary care intervals for patients with stage IV disease. Even though the causal pathway of this finding deserves further exploration, it supports the findings of Tørring et al, and supports the evidence for potential gain from reducing the time to referral in primary care.

\section{Implications for Research and Practice}

A relatively long time to, and sometimes delayed, referral in primary care was mainly seen in patients in whom cancer suspicion was lower, due to 1 or more factors that contributed to a lower risk profile. This can be considered a direct reflection of a well-functioning primary care system, in which both progress and predictive values of symptoms are used as a diagnostic tool.

We also found that there is potential for reducing time to referral for CRC patients presenting in primary care. Acting upon this potential could reduce delay and potentially improve outcomes for those with the longest durations. This could first be achieved by adequate reinvestigation of recurrent potential cancer-related symptoms or signs, particularly if the alternative explanation becomes less plausible. Also evidence of suboptimal diagnostic follow-up, (eg, not safeguarding follow-up consultations or not reconsidering a hemorrhoid diagnosis in case of persistent rectal blood loss), imply there is room for improvement by enhancing patient compliance and GP proactivity. Our findings also demonstrate the challenges of timely diagnosis of CRC in primary care. It is obvious that high risk symptoms (ie, those with high positive predictive value) such as rectal blood loss and anemia warrant further investigation and that GPs act on those. Less obvious is the outcome of the debate about whether ruling out CRC in every patient with non-red flag symptoms would lead to better patient outcomes. The delicate balance between not wanting to miss cancer and preventing unnecessary referrals and the corresponding burden for patients and health care systems is subject to preference and may differ between patients, cultures, and time periods.

Improving identification and referral of CRC patients for those initially presenting with low risk but not no risk symptoms requires innovations in the GP's diagnostic toolbox. Recent research demonstrates that diagnostic tests, such as the fecal immunochemical test for hemoglobin and the calprotectin point-of-care test, may support the diagnostic process of the GP in lower abdominal complaints. ${ }^{20}$ The effectiveness of these and other tests in actual primary care practice, however, needs to be confirmed.

To read or post commentaries in response to this article, see it online at http://www.AnnFamMed.org/content/17/5/419.

Key words: colorectal cancer; delayed diagnosis; early diagnosis; general practice; primary health care

Submitted August 27, 2018; submitted, revised, March 29, 2019; accepted April 11, 2019.

Funding support: This study was funded by the Dutch Cancer Society (KWF), project number: UU2014-7116, and The Netherlands Organization for Health Research and Development (ZonMw), project number: 80-83910-98-13031.

Ethical approval: The study was reviewed by the Medical Ethical Committee (METC) of the University Medical Centre Utrecht and judged exempt from review by the reviewing accredited METC (IRB) according to Dutch law. Dutch Civil Law allows the use of electronic health records for research purposes under certain conditions. According to this legislation, obtaining informed consent from patients is not obligatory for this type of observational study.

Previous presentation: Preliminary results of this work were presented orally at the annual Meeting of the Cancer and Primary Care Research International Network (Ca-PRI); April 26-28, 2016; Boston, Massachusetts.

Acknowledgments: The authors thank all clinical researchers involved in the data collection. We thank the registration team of the Netherlands Comprehensive Cancer Organisation (IKNL) for the collection of data for the Netherlands Cancer Registry as well as IKNL staff for scientific advice. We thank the GPs in the Utrecht area participating in the Julius General Practitioners' Network for sharing their anonymized electronic health record data and Julia Velikopolskaia and Nicole Boekema of the JGPN for their assistance in extracting data and the linkage procedures.

Supplemental materials: Available at http://www.AnnFamMed. org/content/17/5/419/suppl/DC1/.

\section{References}

1. Ferlay J, Steliarova-Foucher E, Lortet-Tieulent J, et al. Cancer incidence and mortality patterns in Europe: estimates for 40 countries in 2012. Eur J Cancer. 2013:49(6):1374-1403.

2. Data from the Netherlands Cancer Registry, managed by The Netherlands Comprehensive Cancer Organisation. http://www. cijfersoverkanker.nl. Accessed Mar 23, 2017.

3. Maringe C, Walters S, Rachet B, et al; ICBP Module 1 Working Group. Stage at diagnosis and colorectal cancer survival in six highincome countries: a population-based study of patients diagnosed during 2000-2007. Acta Oncol. 2013;52(5):919-932.

4. Tørring ML, Murchie P, Hamilton W, et al. Evidence of advanced stage colorectal cancer with longer diagnostic intervals: a pooled analysis of seven primary care cohorts comprising 11720 patients in five countries. Br J Cancer. 2017:1-10. doi:10.1038/bjc.2017.236

5. De Angelis R, Sant M, Coleman MP, et al. Cancer survival in Europe 1999-2007 by country and age: results of EUROCARE-5-a population-based study. Lancet Oncol. 2013;2045(13):1-12. 
6. Navarro M, Nicolas A, Ferrandez A, Lanas A. Colorectal cancer population screening programs worldwide in 2016: an update. World J Gastroenterol. 2017;23(20):3632-3642.

7. Helsper CCW, van Erp NNF, Peeters PPHM, de Wit NNJ. Time to diagnosis and treatment for cancer patients in the Netherlands: room for improvement? Eur J Cancer. 2017;87:113-121.

8. Rubin GP, Saunders CL, Abel GA, McPhail S, Lyratzopoulos G, Neal RD. Impact of investigations in general practice on timeliness of referral for patients subsequently diagnosed with cancer: analysis of national primary care audit data. Br J Cancer. 2015;112(4):676-687.

9. Mitchell E, Macdonald S, Campbell NC, Weller D, Macleod U. Influences on pre-hospital delay in the diagnosis of colorectal cancer: a systematic review. Br J Cancer. 2008;98(1):60-70.

10. Din NU, Ukoumunne OC, Rubin G, et al. Age and gender variations in cancer diagnostic intervals in 15 cancers: analysis of data from the UK Clinical Practice Research Datalink. PLoS One. 2015;10(5): e0127717.

11. Brandenbarg D, Groenhof F, Siewers IM, Van Der Voort A, Walter FM. Possible missed opportunities for diagnosing colorectal cancer in Dutch primary care : a multimethods approach. Br J Gen Pract. 2018;68(666):e54-e62.

12. Mounce LTA, Price S, Valderas JM, Hamilton W. Comorbid conditions delay diagnosis of colorectal cancer: a cohort study using electronic primary care records. Br J Cancer. 2017;116(12):1536-1543.

13. Van Hout AMGH, de Wit NJ, Rutten FH, Peeters PHM. Determinants of patient's and doctor's delay in diagnosis and treatment of colorectal cancer. Eur J Gastroenterol Hepatol. 2011;23(11): 1056-1063.
14. Smeets HM, Kortekaas MF, Rutten FH, et al. Routine primary care data for scientific research, quality of care programs and educational purposes: the Julius General Practitioners ' [JGPN]. BMC Health Serv Res. 2018;18(1):735.

15. Weller D, Vedsted P, Rubin G, et al. The Aarhus statement: improving design and reporting of studies on early cancer diagnosis. $\mathrm{Br}$ J Cancer. 2012;106(7):1262-1267.

16. The Netherlands Institute of Social Research. https://www.scp.nl/ Onderzoek/Lopend_onderzoek/A_Z_alle_lopende_onderzoeken/ Statusscores. Accessed Jun 10, 2015.

17. O'Halloran J, Miller GC, Britt H. Defining chronic conditions for primary care with ICPC-2. Fam Pract. 2004;21(4):381-386.

18. Macleod U, Mitchell ED, Burgess C, Macdonald S, Ramirez AJ. Risk factors for delayed presentation and referral of symptomatic cancer: evidence for common cancers. Br J Cancer. 2009;101(Suppl 2): S92-S101.

19. Hamilton W. The CAPER studies: five case-control studies aimed at identifying and quantifying the risk of cancer in symptomatic primary care patients. Br J Cancer. 2009;101 Suppl(S2):S80-6. doi:

20. Elias SG, Kok L, de Wit NJ, et al. Is there an added value of faecal calprotectin and haemoglobin in the diagnostic work-up for primary care patients suspected of significant colorectal disease? A crosssectional diagnostic study. BMC Med. 2016;14(1):141.

\section{CORRECTIONS}

Ann Fam Med 2019;17:427. https://doi.org/10.1370/afm.2459.

In: Adler RN, Ferguson WJ, Antar H, et al. Transformation support provided remotely to a national cohort of optometry practices. Ann Fam Med. 2019;17(Suppl_1):S33-S39, the authors intended to thank Sai Cherala, $\mathrm{MD}, \mathrm{MPH}$ for her contributions to establishing the cost estimate for avoided ED visits. The online version of the paper, at http://www.annfammed.org/content/17/Suppl_1/S33, now includes the acknowledgment, so the online version differs from the print. The authors regret the omission.

Ann Fam Med 2019;17:427. https://doi.org/10.1370/afm.2460.

In: Lichkus J, Liaw WR, Phillips RL. Utilizing PHATE: a population health-mapping tool to identify areas of food insecurity. Ann Fam Med. 2019;17(4):372-372, the phrase, "Can change to" was extraneous and the sentence, "Many physicians, however, lack the tools needed to effectively address them" was repeated twice; both should have been deleted during the copy editing process. The error has been corrected in the online version at http://www.annfammed.org/content/17/4/372, therefore the print version differs from the online version. The publisher regrets the error. 